\title{
Detection of microbes, aflatoxin and toxic heavy metals in Chinese medicinal herbs commonly consumed in Thailand
}

W. Rangsipanuratn ${ }^{l}$, P. Kammarnjassadakul ${ }^{1}$, I. Janwithayanuchit ${ }^{l}$, P. Paungmoung ${ }^{l}$, S. Ngamurulert ${ }^{1}$, M. Sriprapun ${ }^{2}$, S. Yangen ${ }^{3}$, V. Soottitantawat ${ }^{4}$, A. Sandee ${ }^{* 5}$

${ }^{I}$ Faculty of Medical Technology, Huachiew Chalermprakiet University, Samutprakarn, Thailand.

${ }^{2}$ Department of Microbiology, Faculty of Pharmacy, Mahidol University, Thailand.

${ }^{3}$ Faculty of Public and Environmental Health, Huachiew Chalermprakiet University, Samutprakarn, Thailand.

${ }^{4}$ Faculty of Chinese Medicine, Huachiew Chalermprakiet University, Samutprakarn, Thailand.

${ }^{5}$ Chulabhorn Research Institute, Bangkok, Thailand

\begin{abstract}
The increasing popularity of Chinese herbal treatment has sparked interest in investigating how safe these alternative medicines are. This study scrutinized the Chinese herbs used most popularly in Thailand to identify the presence of heavy metals, microorganisms and aflatoxin contamination. Five commonly consumed Chinese herbs were investigated: Milkvetch root (Astragalus membranaceus), Chinese angelica (Angelica sinensis), Goji berry (Lycium barbarum), Chinese yam (Dioscorea opposita) and Jujube (Ziziphus jujuba). Any heavy metal (arsenic, lead, cadmium), microorganisms (total viable count; total aerobic microbial count, total combined yeast and mold count), pathogenic bacteria (Staphylococcus aureus, Salmonella spp., Clostridium spp.) or aflatoxin contamination were examined. Of the 25 samples, sixteen (64.0\%) did not exceed acceptable limits. Nor did the total aerobic microbial count, total combined yeast and mold count, aflatoxin and heavy metals exceed permissible limits. Neither Salmonella spp., nor S. aureus were found in any of the samples. However, significant Clostridium spp. contamination (36.0\%) was detected in 9 of the 25 samples. These health hazards should be addressed systematically and for the long term by the appropriate consumer protection agencies and related organizations.
\end{abstract}

Keyword: Chinese medicinal herbs, microbes, aflatoxin, heavy metals

\section{INTRODUCTION}

Medicinal herbs have long been used by mankind for primary health care. It has been reported that about $70-80 \%$ of the world's population depends on medicinal herbs ${ }^{1}$. In developing countries as many as $89 \%$ of the people rely on traditional herbal remedies ${ }^{2}$. In Chinese communities, medicinal herbs are widely used for the treatment of various illnesses. Chinese medicinal herbs in particular are frequently resorted to as alternative treatments in cases where commercial pharmaceuticals fail ${ }^{3}$.

While interest in the therapeutic use of herbal products continues to grow, scientific certainty about the safe and salutary properties of Chinese medicinal herbs has been lacking ${ }^{4}$. The increasing consumption of medicinal herbs has led to public health problems where effective surveillance has not monitored the use, efficacy, toxicity and quality of these products. In addition, investigations tend to take place only when cases of toxic results are reported ${ }^{5}$. There is a widespread misconception that consuming herbs and plants is naturally and inherently safe. Recently, however, news of adverse, even toxic, effects linked to the use of herbs and herbal formulations has drawn greater attention ${ }^{6}$. Medicinal herbs become toxic when they are contaminated with microbes, heavy 
metals, pesticides, and other harmful chemicals ${ }^{4}$. The eight common heavy metal pollutants listed by the EPA (Environment Protection Agency) are cadmium, arsenic, chromium, copper, nickel, mercury, lead and zinc ${ }^{7}$. The toxic impact of heavy metals in the environment and on human health has attracted considerable attention in recent years. Heavy metals have a tendency to accumulate in the food chain. Plants growing in contaminated soil become the main avenues of transfer of these dangers, whether directly or indirectly, to humans. Heavy metals ingested can accumulate in the bodies of humans. When the level of intake reaches a certain stage, the results are poisonous, and serious health problems can occur, for example, renal failure, liver damage and symptoms of chronic toxicity ${ }^{8}$.

Microbial contamination of medicinal herbs can happen through improper harvesting, handling, storage, packaging and distribution. The most likely source of microbial contamination is from the ground and in processing facilities (contaminated air, microbes of human origin) ${ }^{9}$. Many kinds of microorganisms have been identified in herbal products, for example, Bacillus spp., Staphylococcus spp., Acinetobacter baumannii, Escherichia coli, Enterococcus faecalis, Aspergillus niger, Psedomonas aeruginosa, Clostridium spp. ${ }^{10-13}$. Ingestion of some of these microbial contaminants in herbal products may cause serious health problems.

Besides heavy metals and microbial contaminants, contamination by aflatoxins in medicinal herbs has also been reported ${ }^{14}$. Aflatoxins are mycotoxins produced mainly by Aspergillus parasiticus and Aspergillus flavus and, though rarely, by Aspergillus nomius. Aflatoxins are well known as one of the most powerful carcinogens and mutagens. Other toxic effects of aflatoxins includeimmunosuppression, teratogenicity and genotoxicity. Its contamination in foodstuff and animal feed is controlled by legal limits. The accepted range of total aflatoxins in foodstuffs and in animal feed are 1-20 ppb and 0-50 $\mathrm{ppb}$, respectively ${ }^{14}$. However, the maximum permissible levels of aflatoxin B1 (AFB1) and total aflatoxins in herbal drugs are 2 and $4 \mu \mathrm{g} /$ $\mathrm{Kg}$, respectively ${ }^{15}$. Therefore, increased con- sumption of herbal medicines could lead to greater intake of aflatoxins and the likelihood of more serious health problems.

At present, there is little information available about the biosafety of medicinal herbs sold in Thailand. We have developed this work to contribute to better safety and quality of the herbals commercially available in the Thai market. This study aimed to examine the pathogenic microorganisms, aflatoxins and heavy metals in five commonly consumed Chinese medicinal herbs from five different local shops in Thailand. The report will be used to better inform considerations of the potential risks for human health associated with the consumption of Chinese medicinal herbs.

\section{MATERIALS AND METHODS}

\subsection{Chinese medicinal herbs}

In 2014, 25 samples of five commonly consumed Chinese medicinal herbs - (Milkvetch root, Chinese angelica, Goji berry, Chinese yam, and Jujube) - were purchased anonymously from five different herbal suppliers in Bangkok.

\subsection{Quantification of heavy metal}

Each sample was dried at $70-80^{\circ} \mathrm{C}$ for 2 days and then ground into powder. Twenty milliliters of concentrated nitric acid were added to each 1.5 grams of ground powder and then heated at $70-80^{\circ} \mathrm{C}$ until the remaining volume was less than $5 \mathrm{ml}$. The mixtures were transferred to $50 \mathrm{ml}$ volumetric flasks and adjusted with de-ionized water. The herbal solutions were analyzed for arsenic with Agilent ICP-MS 7500c (Agilent Technologies Inc., USA) using In-house method TE-CH-134 based on AOAC (2012), 986.15 ${ }^{16}$. For lead and cadmium, samples were analyzed using the Thermo Scientific iCE 3500 atomic absorption spectroscopy (AAS), (Thermo Fisher Scientific Inc., USA) $)^{17}$.

\subsection{Microbiological methods}

Each sample of Chinese medicinal herbs was cut into small pieces with sterile scissors. The total viable count of microbial contaminants 
was determined following WHO 2011 Quality control methods for herbal material ${ }^{18}$.

\subsubsection{Total aerobic microbial count (TAMC)}

Ten grams of each sample were diluted at 1:10 in Soybean-Casein Digest broth and then serial dilution was performed until the dilution reached 1: $10^{6}$. Each dilution $(0.1 \mathrm{ml})$ was taken and cultured by spread plate method on Soybean-Casein Digest agar and incubated at $35 \pm 2^{\circ} \mathrm{C}$ for 48 hours. The plate with around 30-300 colonies was chosen for the colony count and the number of the total colony count was multiplied by the dilution factor.

\subsubsection{Total combined yeast and mold count (TYMC)}

Ten grams of each sample were diluted at 1:10 in Sabouraud dextrose broth. Serial dilution was then performed until a dilution of 1: $10^{6}$ was obtained. Each dilution $(0.1 \mathrm{ml})$ was taken and cultured by spread plate method on Sabouraud dextrose agar and incubated at $25-30^{\circ} \mathrm{C}$ for 5-7 days. The plate with about 30300 colonies was chosen for the colony count and the number of the total colony count was multiplied by the dilution factor.

\subsection{Characterization of microbial contaminants}

\subsubsection{Staphylococcus aureus}

Ten grams of each sample were mixed in $100 \mathrm{ml}$ of phosphate buffer saline. Ten milliliters were transferred into trypticase soy broth and incubated at $35 \pm 2^{\circ} \mathrm{C}$ for $18-24$ hours. The cultivated bacteria were sub-cultured into mannitol salt agar (MSA) and incubated at $35 \pm 2^{\circ} \mathrm{C}$ for $18-24$ hours. Isolated colonies of bacteria were identified by morphological appearance and standard biochemical tests ${ }^{19}$.

\subsubsection{Salmonella spp.}

Ten grams of each sample were mixed in $100 \mathrm{ml}$ of trypticase soy broth (TSB) and incubated at $35 \pm 2^{\circ} \mathrm{C}$ for $18-24$ hours. Then $1 \mathrm{ml}$ of cultivated bacteria were sub-cultured into $10 \mathrm{ml}$ of Gram-negative broth (GN) and incubated at $35^{\circ} \mathrm{C}$ for $18-24$ hours. After incubation, the cultivated bacteria was sub-cultured onto
Salmonella-Shigella (SS) agar and incubated at $35 \pm 2^{\circ} \mathrm{C}$ for $18-24$ hours. It was also subcultured into modified semi-solid Rappaport Vassiliadis (MSRV) medium and then incubated at $42^{\circ} \mathrm{C}$ for $18-24$ hours. Isolated colonies of bacteria were identified by morphological appearance and standard biochemical tests ${ }^{19}$.

\subsubsection{Clostridium spp.}

Ten grams of each sample were resuspended in two bottles of $100 \mathrm{ml}$ of cooked meat medium. One bottle was incubated at $100^{\circ} \mathrm{C}$ for 10 minutes and another was incubated at $65^{\circ} \mathrm{C}$ for 30 minutes. The bottles were then sealed with paraffin oil and incubated at $35 \pm$ $2^{\circ} \mathrm{C}$ for $48-72$ hours. Only the bottles containing gram-positive bacilli were chosen for anaerobic culture and identification of Clostridium spp.

\subsection{Determination of aflatoxin}

Twenty grams of each sample were mixed with $70 \%$ methanol, vortexed for 3 minutes, and then left at room temperature for 5-10 minutes. The clear upper parts were filtered and diluted at 1:3 with a washing buffer. Aflatoxin was examined by using the ScreenEZ-Aflatoxin ELISA Test Kit based on Direct Competitive enzyme linked immunosorbent assay.

\section{RESULTS}

The qualitative analysis examined 25 samples of five types of Chinese medicinal herbs (Milkvetch root, Chinese angelica, Goji berry, Chinese yam, and Jujube) randomly collected from five suppliers in Bangkok. All the samples were analyzed for the heavy metals cadmium, lead and arsenic. The results are given in Tables 1-3. The values listed are averages from 3 determinations. The results show that all 25 samples were contaminated with heavy metals such as arsenic, cadmium, and lead, but within permissible levels.

\subsection{Heavy metals contamination}

Arsenic was detected at a level of ND-0.314 ppm. The highest levels of arsenic contamination in these herbals - an average 
$0.125 \mathrm{ppm}$ - were found in Chinese angelica (Table 1). The level of cadmium contamination was ND-0.1517 ppm. The highest levels were found in Chinese yam and Jujube, 0.0389 and $0.0373 \mathrm{ppm}$, respectively (Table 2). Results for analyses of the amount of lead contaminant in Chinese medicinal herbs showed the presence of lead at levels of 0.018 to $4.707 \mathrm{ppm}$. The highest level was found in Milkvetch root with an average of $2.236 \mathrm{ppm}$, followed by Chinese angelica and Chinese yam with an average of 2.154 and 2.147 ppm, respectively (Table 3). Of the 3 metals assayed, lead had the highest concentration (0.018-4.707 ppm).

Table 1. The amount of arsenic (ppm) determined in five types of Chinese medicinal herbs

\begin{tabular}{lccccc}
\hline \multirow{2}{*}{ Chinese herbal medicine } & \multicolumn{5}{c}{ Arsenic (ppm) } \\
\cline { 2 - 6 } & Shop\# 1 & Shop\# 2 & Shop\# 3 & Shop\# 4 & Shop\# 5 \\
\hline 1. Milkvetch root & ND & ND & 0.218 & ND & ND \\
2. Goji berry & 0.185 & ND & ND & ND & ND \\
3. Chinese angelica & ND & ND & 0.185 & 0.217 & 0.225 \\
4. Chinese yam & ND & ND & 0.314 & ND & ND \\
5. Jujube & ND & ND & ND & ND & 0.185 \\
\hline
\end{tabular}

$\mathrm{ND}=$ not detected (less than detection limit; $0.145 \mathrm{ppm}$ )

Table 2. The amount of cadmium (ppm) determined in five types of Chinese medicinal herbs

\begin{tabular}{lccccc}
\hline \multirow{2}{*}{ Chinese herbal medicine } & \multicolumn{5}{c}{ Cadmium (ppm) } \\
\cline { 2 - 6 } & Shop\# 1 & Shop\# 2 & Shop\# 3 & Shop\# 4 & Shop\# 5 \\
\hline 1. Milkvetch root & ND & ND & ND & ND & ND \\
2. Goji berry & 0.1517 & ND & ND & 0.0001 & 0.0004 \\
3. Chinese angelica & ND & ND & ND & ND & 0.0052 \\
4. Chinese yam & 0.0496 & ND & 0.1439 & ND & 0.0014 \\
5. Jujube & 0.0655 & 0.1202 & ND & ND & 0.0008 \\
\hline
\end{tabular}

$\mathrm{ND}=$ not detected (less than detection limit; $0.01 \mathrm{ppb})$

Table 3. The amount of lead (ppm) determined in five types of Chinese medicinal herbs

\begin{tabular}{lccccc}
\hline \multirow{2}{*}{ Chinese herbal medicine } & \multicolumn{5}{c}{ Lead (ppm) } \\
\cline { 2 - 6 } & Shop\# 1 & Shop\# 2 & Shop\# 3 & Shop\# 4 & Shop\# 5 \\
\hline 1. Milkvetch root & 2.971 & 3.765 & 4.284 & 0.143 & 0.019 \\
2. Goji berry & 2.281 & 3.903 & 3.991 & 0.019 & 0.018 \\
3. Chinese angelica & 3.091 & 2.924 & 4.707 & 0.021 & 0.018 \\
4. Chinese yam & 3.196 & 4.209 & 3.132 & 0.179 & 0.018 \\
5. Jujube & 2.804 & 3.343 & 3.017 & 0.020 & 0.018 \\
\hline
\end{tabular}




\subsection{Pathogenic bacteria contamination}

All 25 samples were tested for the presence of pathogenic bacteria, including Staphylococcus aureus, Salmonella spp., and Clostridium spp., and aflatoxin. Table 4 shows that these samples of Chinese medicinal herbs were contaminated with aerobic microbes, but at acceptable levels. The total aerobic microbial count (TAMC) detected in all of the 5 samples of Milkvetch roots and Goji berry from 5 different shops showed that 100 percent were contaminated. The 4 samples of Jujube from 5 different shops show that 80 percent were contaminated. At the same time 40 percent, or 2 of the 5 samples of Chinese angelica and Chinese yam, were contaminated.

Table 4. Total aerobic microbial count (CFU/g) in five types of Chinese medicinal herbs

\begin{tabular}{lccccc}
\hline \multirow{2}{*}{ Chinese herbal medicine } & \multicolumn{5}{c}{ TAMC* } \\
\cline { 2 - 6 } & Shop\# 1 & Shop\# 2 & Shop\# 3 & Shop\# 4 & Shop\# 5 \\
\hline 1. Milkvetch root & $1.9 \times 10^{3}$ & $9.0 \times 10^{3}$ & $4.5 \times 10^{2}$ & $2.0 \times 10^{2}$ & $2.5 \times 10^{2}$ \\
2. Goji berry & $4.0 \times 10^{2}$ & $3.5 \times 10^{2}$ & $7.6 \times 10^{3}$ & $5.5 \times 10^{2}$ & $6.5 \times 10^{2}$ \\
3. Chinese angelica & TLTC & TLTC & TLTC & $3.8 \times 10^{2}$ & $4.5 \times 10^{2}$ \\
4. Chinese yam & TLTC & TLTC & TLTC & TLTC & TLTC \\
5. Jujube & $4.7 \times 10^{3}$ & $2.0 \times 10^{3}$ & TLTC & $3.6 \times 10^{4}$ & $3.5 \times 10^{2}$ \\
\hline
\end{tabular}

* According to Thai pharmacopoeia criteria for medicinal herbs, TYMC must not exceed $\leq 5 \times 10^{7} \mathrm{CFU} / \mathrm{g}$ TLTC (too low to count)

For total combined yeast and mold count (TYMC), the highest numbers were found in Milkvetch root and Jujube (40\%). Golgi berry was also found to be contaminated with TYMC (20\%). However, no TYMC was detected in Chinese angelica and Chinese yam, as shown in Table 5.

No contamination of Salmonella spp. or $S$. aureus was found in any of the samples of Chinese herbs $(25 / 25)$, but they were all contaminated with Clostridium spp., found mostly in Milkvetch root (60\%), followed by Chinese angelica and Jujube (40\%), as shown in Table 6. Of 9 samples contaminated with Clostridium spp., 8 were identified as $C$. perfringens and 1 was Clostridium spp. (Table 6).

Table 5. Total yeast and mould counts (CFU/g) in five types of Chinese medicinal herbs

\begin{tabular}{lccccc}
\hline \multirow{2}{*}{ Chinese herbal medicine } & \multicolumn{5}{c}{ TYMC* } \\
\cline { 2 - 6 } & Shop\# 1 & Shop\# 2 & Shop\# 3 & Shop\# 4 & Shop\# 5 \\
\hline 1. Milkvetch root & TLTC & $1 \times 10^{2}$ & $1 \times 10^{2}$ & TLTC & TLTC \\
2. Goji berry & TLTC & TLTC & $5 \times 10^{2}$ & TLTC & TLTC \\
3. Chinese angelica & TLTC & TLTC & TLTC & TLTC & TLTC \\
4. Chinese yam & TLTC & TLTC & TLTC & TLTC & TLTC \\
5. Jujube & TLTC & $8.7 \times 10^{3}$ & TLTC & TLTC & $4.0 \times 10^{3}$ \\
\hline
\end{tabular}

* According to Thai pharmacopoeia criteria for medicinal herbs, TYMC must not exceed $\leq 5 \times 10^{4} \mathrm{CFU} / \mathrm{g}$ TLTC (too low to count) 
Table 6. Examination of Clostridium spp. in five types of Chinese medicinal herbs

\begin{tabular}{lcccccc}
\hline \multirow{2}{*}{ Chinese herbal medicine } & \multicolumn{4}{c}{ Clostridium spp. } & \multicolumn{1}{c}{ Positive (\%) } \\
\cline { 2 - 7 } & Shop\# 1 & Shop\# 2 & Shop\# 3 & Shop\# 4 & Shop\# 5 \\
\hline 1. Milkvetch root & + & + & + & - & - & $60.0(3 / 5)$ \\
2. Goji berry & + & - & - & - & - & $20.0(1 / 5)$ \\
3. Chinese angelica & - & - & + & - & + & $40.0(2 / 5)$ \\
4. Chinese yam & + & - & - & - & - & $20.0(1 / 5)$ \\
5. Jujube & - & + & + & - & - & $40.0(2 / 5)$ \\
Positive (\%) & $60.0(3 / 5)$ & $40.0(2 / 5)$ & $60.0(3 / 5)$ & $0(0 / 5)$ & $20.0(1 / 5)$ \\
\hline
\end{tabular}

\subsection{Aflatoxin contamination}

Aflatoxin contamination was observed in Chinese medicinal herbs. Contaminants have been found in all samples, but not in excess of the acceptable levels prescribed by the Ministry of Public Health, Thailand. Comparing the five kinds of Chinese herbal medicines,
Chinese angelica was the most contaminated with aflatoxin with average of $9.87 \mathrm{ppb}$, followed by Milkvetch root and Jujube with an average of 4.23 and $4.21 \mathrm{ppb}$, respectively. Of these, one sample of Chinese angelica showed the highest aflatoxin contaminant level of $19.65 \mathrm{ppb}$ (Table 7).

Table 7. The amount of aflatoxin in five types of Chinese medicinal herbs

\begin{tabular}{lccccc}
\hline \multirow{2}{*}{ Chinese herbal medicine } & \multicolumn{5}{c}{ Aflatoxin (ppb) } \\
\cline { 2 - 6 } & Shop\# 1 & Shop\# 2 & Shop\# 3 & Shop\# 4 & Shop\# 5 \\
\hline 1. Milkvetch root & 2.42 & 3.35 & 3.74 & 5.57 & 6.06 \\
2. Goji berry & 1.62 & 1.76 & 1.96 & 4.56 & 5.78 \\
3. Chinese angelica & 5.68 & 4.72 & 6.11 & 19.65 & 13.18 \\
4. Chinese yam & 1.64 & 2.11 & 2.44 & 4.79 & 4.83 \\
5. Jujube & 2.63 & 3.19 & 2.49 & 6.62 & 6.10 \\
\hline
\end{tabular}

\section{DISCUSSION}

At present, the World Health Organization (WHO) reports that between 70 and 80 percent of the world's population relies on non-conventional medicine - especially Chinese medicinal herbs - for the treatment of disease and as a food supplement ${ }^{1}$. Reflecting the high demand, there are many kinds of Chinese herbal products available on the market. It is becoming clear that many of these herbal products are contaminated with microbes, heavy metals, chemical toxins, and pesticides. The heavy metals may reflect the sources of these herbal materials. The microbial contaminants may be related to the source and also to the handling processes. The toxicity of frequently used Chinese medicines has been shown to affect the liver and the kidneys as well as the nervous, gastrointestinal, and cardiovascular systems ${ }^{20}$. Chinese herbs sold to the general public, whether marketed for humans or animals, should have proven quality, safety and efficacy (QSE). Monitoring of the microbiological contents and levels of heavy metal contamination is very important. This study has attempted to quantify the presence of heavy metals, aerobic 
bacteria, yeast and mold, S. aureus, Salmonella spp., Clostridium spp., and aflatoxin contamination in Chinese medicinal herbs commonly consumed in Thailand. For this study, 25 common samples of Milkvetch root, Goji berry, Chinese angelica, Chinese yam, and Jujube were obtained from five different shops.

The heavy metal content (lead, cadmium, and arsenic) was examined in all 25 samples by atomic absorption spectrophotometer (AAS) using Graphite furnace technique. Heavy metal contaminants were found in different amounts: ND-0.314 ppm of arsenic, ND-0.1517 ppm of cadmium and 0.018-4.707 ppm of lead. All are below the recommended levels according to legal limits of arsenic ( $\leq 4 \mathrm{ppm})$, cadmium $(\leq 0.3 \mathrm{ppm})$, and lead $(\leq 10 \mathrm{ppm})$ in medicinal herbs and herbal products in Thailand. The levels of these toxic metals are comparable to those in previous reports ${ }^{21-24}$, in which the levels of arsenic, cadmium and lead were found not to exceed the limit of $1,0.3$, and 10 ppm, respectively ${ }^{25}$. The Chinese medicinal herbs used in this study were possibly contaminated with heavy metal from an environment. Awareness of the ubiquitous presence of these contaminants in dense urban settings, for example - should be kept in mind when collecting medicinal herbs from cultivated areas which are prone to heavy metal pollution. At the same time, such contamination may occur during open-air drying, preserving or manufacturing processes ${ }^{26,27}$. Numerous case reports originating from countries such as the USA, Singapore, Malaysia, Africa, Belgium, China, and the UK have identified heavy metal contaminants in traditional Chinese medicinal herbs ${ }^{6,28}$. Manganese, copper, cadmium, ferric, and zinc have all been found in samples of four commonly consumed herbal medicines in Malaysia, though generally in low concentrations ( $<1 \mathrm{mg} / \mathrm{L})$ except for manganese $(18.545 \mathrm{mg} / \mathrm{L})$. Boiling was found to reduce the copper content but not other metals 5 . The samples in this study may not appear to pose much risk for heavy metal toxicity, but real danger is threatened to those who ingest these traditional remedies too frequently or in too great a quantity. Contamination by heavy metal, even in low concentrations, should be a concern. In the absence of periodic examination of these markets and these products, we must assume that the safety of consumers is at risk.

This study also investigated microbial contaminants. According to Thai pharmacopoeia criteria for medicinal herbs, TAMC and TYMC must not exceed $5 \times 10^{7} \mathrm{CFU} / \mathrm{g}$ and $5 \times 10^{4} \mathrm{CFU} / \mathrm{g}$, respectively. In addition, the product should not contain any pathogenic bacteria. The present study found that all the Chinese herbs studied contained, TAMC and TYMC, though within permissible limits. No contamination of pathogenic bacteria, S. aureus and Salmonella spp. was found. However, all five types (9/25 samples/ $36 \%$ ) of Chinese herbs were found to be contaminated with Clostridium spp., mostly Clostridium perfringens. Clostridium spp. is commonly found in the ground, and the spores can exist in the environment for a long time. Furthermore, some secondary metabolites produced by Clostridium spp. can be toxic and can cause health problems in humans. In particular, Clostridium perfringens type A enterotoxin is known to cause diseases of the gastrointestinal tract. Hence, a person who has diarrhea due to food poisoning could actually get worse after taking doses of tainted herbal medicine. Clostridium spp. contamination is also dangerous because the spores of Clostridium botulinum can cause infant botulism. The use of herbals teas as a natural remedy against intestinal colic must be avoided in children younger than one year. Our present study is consistent with many previous reports. Bianco et al. found that $7.5 \%$ of 200 samples of chamomile tea were contaminated with the spores of $C$. botulinum $^{29}$. Clostridium spores were also found in other medicinal herbs. In one investigation, Clostridium perfringens spores were found in $83.9 \%$ of the investigated herbal samples ${ }^{30}$. To avoid microbial contaminants and other toxins in herbal medicine, gamma-irradiation or drying at high temperature should be performed ${ }^{9}$.

Chinese medicinal herbs distributed in Thailand take many forms. There are raw medicinal herbs, pills, powders, capsules containing powder, Infusions, hot teas, and herbal drugs preserved in alcohol. On the other hand, there are many ways to decontaminate herbal medicines, depending on their form. 
For Chinese herbal decoctions, debris or dust can be removed by soaking in water about 30 minutes, then boiling for 10 minutes. This method decontaminates bacteria without spores. To destroy proteolytic C. botulinum spore, heating at $121.1^{\circ} \mathrm{C}$ for 3 minutes or at $85^{\circ} \mathrm{C}$ for 52 minutes should be performed ${ }^{31}$. The botulinum toxin itself, as opposed to the bacterium, can be destroyed by heating at $85^{\circ} \mathrm{C}$ for at least 5 minutes ${ }^{32}$. The vegetative cells and spores of $C$. perfringens type A1, a heat-sensitive strain, can be destroyed by boiling for just 2-3 minutes. However, spores of the $\mathrm{A} 2$ and $\mathrm{C}$ type are more heat-resistant and must be boiled at $100^{\circ} \mathrm{C}$ for $30-60$ minutes

Raw materials to be transformed into powder, pills, or capsules of Chinese medicinal herbs should be decontaminated by rinsing with water for at least 10 minutes and dried under the sun or heat-dried at a temperature of $50-60^{\circ} \mathrm{C}$, then blended, or crushed into powder. Finally, the raw materials should be further decontaminated by dry heat, moist heat, baking with ethylene oxide gas or ozone gas, heating in a microwave, or using Ultra-violet light or gamma rays. Each method has advantages and disadvantages and results, to some extent, in the loss of the drug's active ingredients. In the preparation of raw materials for the manufacture of herbal tea, soaking in boiling water is not enough to destroy spores of Clostridium spp. Manufacturers should get rid of debris, dust and other contaminants from raw herbs before performing the next steps.

Aflatoxin is a toxic metabolite. It is produced by Aspergillus flavus (A. flavus) and A. parasiticus, which can be found throughout the environment. The toxins are heat tolerant up to $240-289^{\circ} \mathrm{C}^{33}$ and cannot be disintegrated by decoction or even after prolonged boiling. The Chinese medicinal herbs discussed here are all made from roots, rhizomes, and the ripe fruit of herbal plants. The study found that all the samples were contaminated with aflatoxin, though within permissible limits $(<20 \mathrm{ppb})^{34}$. This result is consistent with several previous studies which also detected aflatoxin contaminants in Chinese herbs and the most commonly used roots, rhizomes and ripe fruits ${ }^{35}$.
One of the samples of Chinese angelica was contaminated with aflatoxin (19.65 ppb) to a degree almost exceeding the permissible maximum. In the present study, aflatoxin was found in all the samples. Perhaps soil fungus thrives in the starch and fat of Chinese herbal roots and rhizomes. Preventing fungus contamination in soil could well be the best way to reduce the aflatoxin which is being absorbed by these herbs.

In many countries, medicinal herbs are largely marketed without any oversight from the Ministry of Health because they are considered to be food supplements. In other words, they are not held to pharmaceutical standards of quality. Both this present study and many earlier ones strongly recommend that quality controls on medicinal herbs be tightened and upgraded. To overcome health problems caused by the contamination of microbes and heavy metals, there should be measures stipulating standard operating procedures (SOP) at source. Good Laboratory Practice (GLP) and Good Manufacturing Practice (GMP) guides are also needed to produce safe, good quality, Chinese medicinal herbs.

These findings have implications in the matter of medicinal herbs for human consumption. They provide additional data on contamination by microbes and heavy metals in products consumed by many Thai people. At the very least, herbal materials should be harvested from unpolluted and uncontaminated sources. Effective quality control of medicinal herbs is vital if the toxic effects of long term use and overconsumption are to be prevented.

\section{ACKNOWLEDGMENTS}

This research was financially supported by Huachiew Chalermprakiat University. We thank Janice M.Wongsurawat, an English language consultant at CRI in the Academic Office, for editing the manuscript.

\section{REFERENCES}

1. Akerele O. Nature's medicinal bounty: don't throw it away. World Health Forum. 1993;14:390-5. 
2. Shaw D. Risks or remedies? Safety aspects of herbal remedies in the UK. J R Soc Med. 1998;91:294-6.

3. Atherton DJ, Sheehan MP, Rustin MH, Whittle B, Guy G. Treatment of atopic eczema with traditional Chinese medicinal plants. Pediatr Dermatol. 1992;9:373-5.

4. Saad B, Azaizeh H, Abu-Hijleh G, Said O. Safety of traditional arab herbal medicine. Evid Based Complement Alternat Med. 2006;3:433-9.

5. Ting A, Chow Y, Tan W. Microbial and heavy metal contamination in commonly consumed traditional Chinese herbal medicines. J Tradit Chin Med. 2013;33: 119-24.

6. Ernst E. Toxic heavy metals and undeclared drugs in Asian herbal medicines. Trends Pharmacol Sci. 2002;23:136-9.

7. Arthar MVS. Heavy metals and environment. New Delhi: New international Wiley Eastern ;1995.

8. Korfali SI, Hawi T, Mroueh M. Evaluation of heavy metals content in dietary supplements in Lebanon. Chem Cent J. 2013;7:10.

9. Kosalec I, Cvek J, Tomic S. Contaminants of medicinal herbs and herbal products. ArhHigRadaToksikol.2009;60:485-501.

10. Nakano H, Okabe T, Hashimoto H, Sakaguchi $\mathrm{G}$. Incidence of Clostridium botulinum in honey of various origins. Jpn J Med Sci Biol. 1990;43:183-95.

11. Schocken-Iturrino RP, Carneiro MC, Kato E, Sorbara JO, Rossi OD, Gerbasi LE. Study of the presence of the spores of Clostridium botulinum in honey in Brazil. FEMS ImmunolMed Microbiol. 1999;24:379-82.

12. Wilson C, Dettenkofer M, Jonas D, Daschner FD. Pathogen growth in herbal teas used in clinical settings: a possible source of nosocomial infection? Am J Infect Control. 2004;32:117-9.

13. Brown JC, Jiang X. Prevalence of antibioticresistant bacteria in herbal products. $\mathrm{J}$ Food Prot. 2008;71:1486-90.

14. Ashiq S, Hussain M, Ahmad B. Natural occurrence of mycotoxins in medicinal plants: a review. Fungal Genet Biol. 2014 ;66:1-10.
15. Council of Europe. European Pharmacopoeia. Strasbourg: Council of Europe; 2011

16. AOAC INTERNATIONAL official methods of analysis online [document on the Internet]; 2005. Available from: http://www.eoma. aoac.org/.

17. American Water Works Association, Water Environment Federation. Standard methods for the examination of water and wastewater. American Public Health Association; 1999.

18. WHO. Quality control methods for herbal materials. Updated edition of Quality control methods for medicinal plant materials, edn. Geneva: WHO Press; 2011.

19. Tille PM. Bailey \& Scott's Diagnostic Microbiology. 13th ed. St. Louis: Elsevier; 2014.

20. Chan K. Some aspects of toxic contaminants in herbal medicines. Chemosphere. 2003 ;52:1361-71.

21. Basgel S, Erdemoglu SB. Determination of mineral and trace elements in some medicinal herbs and their infusions consumed in Turkey. Sci Total Environ. 2006;359:82-9.

22. Maiga A, Diallo D, Bye R, Paulsen BS. Determination of some toxic and essential metal ions in medicinal and edible plants from Mali. J Agric Food Chem. 2005;53: 2316-21.

23. Arpadjan S, Celik G, Taskesen S, Gucer S. Arsenic, cadmium and lead in medicinal herbs and their fractionation. Food Chem Toxicol. 2008;46:2871-5.

24. Zhu F, Wang X, Fan W, Qu L, Qiao M, Yao S. Assessment of potential health risk for arsenic and heavy metals in some herbal flowers and their infusions consumed in China. Environ Monit Assess. 2013;185: 3909-16.

25. WHO. Monographs on selected medicinal plants. Geneva: WHO Press; 1999.

26. Kneifel W, Czech E, Kopp B. Microbial contamination of medicinal plants-a review. Planta Med. 2002;68:5-15.

27. Koh HL, Woo SO. Chinese proprietary medicine in Singapore: regulatory control 
of toxic heavy metals and undeclared drugs. Drug Saf. 2000;23:351-62.

28. Ernst E, Thompson Coon J. Heavy metals in traditional Chinese medicines: a systematic review. Clin Pharmacol Ther. 2001;70: 497-504.

29. Bianco MI, Luquez C, Jong LI, Fernandez RA. Presence of Clostridium botulinum spores in Matricaria chamomilla (chamomile) and its relationship with infant botulism. Int J Food Microbiol. 2008;121:357-60.

30. Martins HM, Martins ML, Dias MI, Bernardo F. Evaluation of microbiological quality of medicinal plants used in natural infusions. Int J Food Microbiol. 2001 15;68:149-53.

31. Peck MW. Clostridium botulinum and the safety of minimally heated, chilled foods: an emerging issue? J Appl Microbiol. 2006 ;101:556-70.
32. Sobel J, Tucker N, Sulka A, McLaughlin J, Maslanka S. Foodborne botulism in the United States, 1990-2000. Emerg Infect Dis. 2004;10:1606-11.

33. Wogan GN. Chemical nature and biological effects of the aflatoxins. Bacteriol Rev. 1966;30:460-70.

34. Tassaneeyakul W, Razzazi-Fazeli E, Porasuphatana S, Bohm J. Contamination of aflatoxins in herbal medicinal products in Thailand. Mycopathologia. 2004;158: 239-44.

35. Han Z, Zheng Y, Luan L, Cai Z, Ren Y, $\mathrm{Wu}$ Y. An ultra-high-performance liquid chromatography-tandem mass spectrometry method for simultaneous determination of aflatoxins B1, B2, G1, G2, M1 and M2 in traditional Chinese medicines. Anal Chim Acta. 2010;664:165-71. 